\title{
Transforming Growth Factor- $\beta$ Is an Upstream Regulator of Mammalian Target of Rapamycin Complex 2-Dependent Bladder Cancer Cell Migration and Invasion
}

Sounak Gupta, ${ }^{*}$ Andrew M. Hau, ${ }^{*}$ Hikmat A. Al-Ahmadie, ${ }^{\dagger}$ Jyoti Harwalkar, ${ }^{\ddagger}$ Aaron C. Shoskes, ${ }^{\ddagger}$ Paul Elson, ${ }^{\S}$ Jordan R. Beach, George S. Hussey," William P. Schiemann, ${ }^{* *}$ Thomas T. Egelhoff, "Philip H. Howe, and Donna E. Hansel*

From the Department of Pathology, * University of California San Diego, La Jolla, California; the Department of Pathology, ${ }^{\dagger}$ Memorial Sloan Kettering Cancer Center, New York, New York; the Departments of Pathology, ${ }^{\ddagger}$ Quantitative Health Sciences, ${ }^{\S}$ and Cellular and Molecular Biology, ${ }^{\text {\ }}$ Cleveland Clinic, Cleveland, Ohio; the Department of Biochemistry," Medical University of South Carolina, Charleston, South Carolina; and the Department of General Medical Sciences-Oncology,** Case Western Reserve University, Cleveland, Ohio

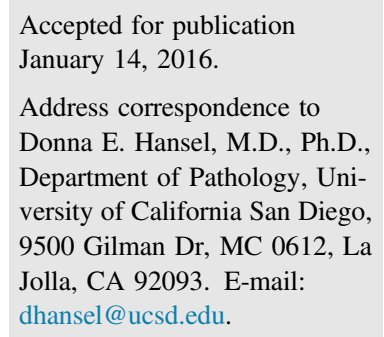

\begin{abstract}
Our prior work identified the mammalian target of rapamycin complex 2 (mTORC2) as a key regulator of bladder cancer cell migration and invasion, although upstream growth factor mediators of this pathway in bladder cancer have not been well delineated. We tested whether transforming growth factor (TGF)- $\beta$, which can function as a promotility factor in bladder cancer cells, could regulate mTORC2-dependent bladder cancer cell motility and invasion. In human bladder cancers, the highest levels of phosphorylated SMAD2, a TGF- $\beta$ signaling intermediate, were present in high-grade invasive bladder cancers and associated with more frequent recurrence and decreased disease-specific survival. Increased expression of TGF- $\beta$ isoforms, receptors, and signaling components was detected in invasive high-grade bladder cancer cells that expressed Vimentin and lacked E-cadherin. Application of TGF- $\beta$ induced phosphorylation of the Ser473 residue of AKT, a selective target of mTORC2, in a SMAD2- and SMAD4-independent manner and increased bladder cancer cell migration in a modified scratch wound assay and invasion through Matrigel. Inhibition of TGF- $\beta$ receptor I using SB431542 ablated TGF- $\beta$-induced migration and invasion. A similar effect was seen when Rictor, a key mTORC2 component, was selectively silenced. Our results suggest that TGF- $\beta$ can induce bladder cancer cell invasion via mTORC2 signaling, which may be applicable in most bladder cancers. (Am J Pathol 2016, 186: 1351-1360; http://dx.doi.org/10.1016/j.ajpath.2016.01.008)
\end{abstract}

Bladder cancer is the fourth leading cause of new cancer diagnoses in men in the United States and represents the seventh leading cause of cancer-related deaths in 2012 in this population. ${ }^{1}$ The high risk of recurrence and progression, coupled with the lack of definitive therapy for many patients, necessitates lifelong surveillance. ${ }^{2}$ Specifically, urothelial carcinoma, which accounts for $>90 \%$ of all bladder cancer cases in the United States, has a poor prognosis once invasion into the deep bladder wall occurs. ${ }^{3}$ Mammalian target of rapamycin complex 2 (mTORC2) has recently emerged as a potential regulator of cancer cell invasion and metastasis, ${ }^{4-6}$ with recent studies from our laboratory showing the importance of this protein complex in driving bladder cancer migration and invasion. ${ }^{7}$ mTORC2 can be activated by upstream phosphoinositide 3-kinase signaling and association with the ribosome, ${ }^{8,9}$ although specific upstream activators of this complex in bladder cancer have not been defined.

Transforming growth factor (TGF)- $\beta$ signaling is complex and has been studied in numerous cancer models as a

\footnotetext{
Supported by NIH grant GM50009 (T.T.E.), National Cancer Institute Case Western Reserve University/Cleveland Clinic Clinical and Translational Science Award (CTSA) grant UL1 RR024989, a KL2 career development award RR024990 (D.E.H.), and the Urology Cares Foundation Research Scholars Award (A.M.H.).

Disclosures: None declared.
} 
promotility agent, as well as a motility repressor. ${ }^{10}$ TGF- $\beta$ isoforms signal through canonical signaling pathways that include activation of SMAD proteins or through noncanonical signaling pathways that include phosphatidylinositol 3kinase, RhoA, or TAK1 pathways. ${ }^{10,11}$ Procancer effects may be driven by either canonical or noncanonical activity, with noncanonical activity shown to promote cancer cell invasion through induction of epithelial-mesenchymal transition (EMT), modulation of miRNA levels, and stimulation of mTOR activity. ${ }^{12-15}$ Recent work has also suggested a role for TGF- $\beta$ in regulating cancer progression through effects on the tumor microenvironment, including immune modulation and effects on cancer-associated fibroblasts. ${ }^{10,11}$

The role of TGF- $\beta$ in bladder cancer has been studied in significantly less detail. It has been shown that several singlenucleotide polymorphisms in genes encoding TGF- $\beta 1$ and the TGF- $\beta$ receptor I (TGF $\beta$ RI) appear to be involved in bladder cancer risk and prognosis. ${ }^{16-18}$ Analysis of the TGF- $\beta$ isoform and receptor expression using immunohistochemistry and PCR has yielded mixed results, likely reflecting the complex biology associated with this signaling pathway in cancer. Although some studies have shown reduced TGF- $\beta$ expression in progressive disease, others have shown increased TGF- $\beta$ expression in higher-grade and higher-stage lesions. ${ }^{19-25}$ In vitro testing has demonstrated that TGF- $\beta$ can induce apoptosis and inhibit tumor growth, but can also increase gene expression associated with tumor progression and can induce matrix metalloproteinase production. ${ }^{26-30}$ These reports suggest that TGF- $\beta$ may perform multiple protumorigenic roles in bladder cancer, although the function of $\mathrm{mTORC} 2$ as a mediator of TGF- $\beta$ activity in this context has not been examined.

Herein, we tested whether TGF- $\beta$ can induce bladder cancer cell motility and invasion through mTORC2-driven signaling. Using a combination of human tumor specimens and in vitro model systems, we show increased TGF- $\beta$ signaling in advanced bladder cancer and TGF- $\beta$-induced bladder cancer cell motility and invasion that is dependent on mTORC2 signaling. The results from this study suggest that TGF- $\beta$ and mTORC 2 activity may function cooperatively in advanced bladder cancer and that targeting of these pathways in combination may be of value in bladder cancer.

\section{Materials and Methods}

\section{Patient Specimens}

Permission for this study was obtained from the Institutional Review Board. Bladder cancer tissue used for immunoblotting consisted of flash-frozen specimens obtained from noninvasive and muscle-invasive carcinoma. Frozen section analysis was performed to ensure that $>90 \%$ of the specimen consisted of tumor. Bladder cancer grade and stage were confirmed by pathology review. Tissue samples were homogenized using the Power Gen 500 (Fisher Scientific, Pittsburgh, PA) on the basis of the manufacturer's instructions. In addition, archived paraffin blocks from patients who underwent biopsy or cystectomy were used for tissue microarray construction and immunohistochemical analysis.

\section{Immunohistochemistry and Patient Outcomes}

Tissue microarray slides were stained on a Discovery XT automated stainer/bench-top $/ 37^{\circ} \mathrm{C}$ incubator using an antigen retrieval solution consisting of Tris/borate/EDTA buffer, $\mathrm{pH}$ 8.0 to 8.5 (Ventana Medical Systems, Tucson, AZ) for 8 minutes at $95^{\circ} \mathrm{C}, 28$ minutes at $100^{\circ} \mathrm{C}$, and 8 minutes at $37^{\circ} \mathrm{C}$. An antibody to phospho-SMAD2 (Ser 465/467) was used at 1:500 dilution (Cell Signaling Technology, Beverly, MA), followed by OmniMap anti-rabbit horseradish peroxidase secondary antibody (Ventana Medical Systems). Slides were counterstained with Hematoxylin II and Bluing Reagent (Ventana Medical Systems). Staining was scored by intensity of nuclear expression. A score of 0 indicates no staining, $1+$ indicates minimal staining, $2+$ indicates moderate staining, and $3+$ indicates intense staining. Statistical analysis was performed using SPSS version 17.0 (SPSS Inc., Chicago, IL) and Statistica version 12.0 (Statsoft Inc., Tulsa, OK). All tests were two-tailed and analyzed at a significance level of $P<0.05$. Outcomes were measured from time of surgery. The end point in survival analysis was disease-related death, measured in time as a function of months from surgery until death. Data were analyzed using proportional hazards models. Patients who died before recurrence were considered failures, but not as a result of their disease; and the Fine and Gray approach was used to account for this competing risk.

\section{Cell Culture and Reagents}

RT4 (ATCC HTB-2), UMUC3 (ATCC CRL-1749), T24 (ATCC HTB-4), and J82 (ATCC HTB-1) cell lines were purchased from the ATCC cell line bank (Manassas, VA). Cells were grown in RPMI 1640 medium (Gibco, Invitrogen Corp., Grand Island, NY) supplemented with $10 \%$ fetal bovine serum (Gibco) and antibiotic/antimycotic solution (Sigma, Inc., St. Louis, MO). All cell lines were maintained at $37^{\circ} \mathrm{C}$ in $5 \% \mathrm{CO}_{2}$. Serum starvation was performed by maintaining cells in serumfree media for 24 hours and washing with sterile phosphatebuffered saline at 6-hour intervals. TGF- $\beta 2$ was a gift from Dr. Philip Howe (Medical University of South Carolina, Charleston, SC) and was applied at $5 \mathrm{ng} / \mathrm{mL}$. SB431542 was obtained from TOCRIS Biosciences (Minneapolis, MN) and used at $2 \mu \mathrm{mol} / \mathrm{L}$.

\section{Immunoblotting}

Cells and tissue samples were extracted in radioimmunoprecipitation assay buffer containing protease inhibitor cocktail (Sigma, Inc.) and phosphatase inhibitor cocktails 1 and 2 (Sigma, Inc.) and subjected to SDSPAGE on $4 \%$ to $15 \%$ gradient gels. Proteins were transferred to Hybond electrochemiluminescence membranes (GE Healthcare Life Sciences, Pittsburgh, PA) using the Bio-Rad Transblot semidry transfer system (Life Science 
Research, Hercules, CA). Membranes were blocked with $1 \%$ bovine serum albumin and incubated with primary antibodies overnight at $4{ }^{\circ} \mathrm{C}$ in blocking solution. The following primary antibodies were purchased from Cell Signaling Technology and used at the following dilutions: Rictor (1:1000), p-AKT (Ser473; 1:1000), pan-AKT (1:2000), p-S6 (1:2000), total-S6 (1:2000), p-SMAD2 (1:1000), N-cadherin (1:1000; clone D4R1H), SMAD2 (1:1000), SMAD4 (1:1000), $\beta$-catenin (1:1000; clone D10A8), Vimentin (1:1000; clone D7D12), TCF8/ZEB1 (1:1000; clone D80D3), zonula occludens protein 1 (1:1000; clone D7D12), Snail (1:1000; clone C15D3), Slug (1:1000; clone C19G7), Claudin-1 (1:1000; clone D5H1D), and $\beta$-actin (1:5000). E-cadherin (1:1000; clone 24E10) and total SMAD2/3 (1:1000) were purchased from BD Biosciences (San Jose, CA). Blots were incubated with alkaline phosphatase-conjugated secondary antibodies (Jackson ImmunoResearch, West Grove, PA) at room temperature for 1 hour and developed using electrochemifluorescence Western blotting reagent packs (GE Healthcare Life Sciences). Densitometry was performed using Imagequant software (GE Healthcare Life Sciences).

\section{PCR}

RNA was extracted using the Qiagen RNAeasy Micro Kit (Qiagen, Valencia, CA) and quantitated by Quant-It RiboGreen RNA Assay Kit (Invitrogen, Grand Island, NY). Reverse transcription was performed using $1 \mu \mathrm{g}$ RNA with qScript cDNA supermix (Quanta Biosciences, Gaithersburg, MD) to generate cDNA. PCR was performed using an annealing temperature of $55^{\circ} \mathrm{C}$ and cycle number of 35 . PCR products were run on $2 \%$ agarose gels. Glyceraldehyde-3-phosphate dehydrogenase (GAPDH) was coamplified with genes of interest as a normalization control. Gel densitometry was performed using ImageQuant TL version 8.1 (GE Healthcare Life Sciences). Primer sequences included the following: TGFBRI, 5'-GATGGGCTCTGCTTTGTCTC-3' (forward) and 5'-TCAAAAAGGGATCCATGCTC-3' (reverse); TGFBRII, 5'-TTTTCCACCTGTGACAACCA-3' (forward) and 5'-GCTGAATGCCTGTCACTTGAA-3' (reverse); SMAD2, 5'-AGCTGGCAGCCTTTGTTTTA- $3^{\prime}$ (forward) and $5^{\prime}$-GTGTGCCTGGGACTTGTTTT$3^{\prime}$ (reverse); SMAD3, 5'-GCCAAATGCTGTGAGTCTGA-3' (forward) and $5^{\prime}$-CATACGCCCAAAGCACCTAT- ${ }^{\prime}$ (reverse); SMAD4, 5'-TGTGTGACACCACCCTCCTA- $3^{\prime}$

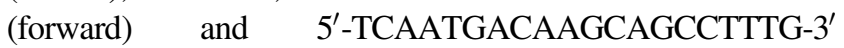
(reverse); and glyceraldehyde-3-phosphate dehydrogenase, 5'-GTCAGTGGTGGACCTGACCT-3' (forward) and 5'-AGGGGTCTACATGGCAACTG-3' (reverse).

\section{Real-Time PCR}

Total RNA from cell cultures was isolated using the NucleoSpin RNA II kit (Macherey-Nagel Inc., Bethlehem, PA), followed by cDNA synthesis using the qScript cDNA Synthesis kit (Quanta Biosciences). TaqMan probes for human TGFRB1 (Hs00610320_m1), TGFRB2
(Hs00234253_m1), SMAD2 (Hs00183425_m1), SMAD3 (Hs00969210_m1), SMAD4 (Hs00929647_m1), and glyceraldehyde-3-phosphate dehydrogenase (Hs02758991_g1), and TaqMan Fast Advanced Master Mix were from Life Technologies, Inc. (Thermo Fisher Scientific, Grand Island, NY). Quantitative RT-PCR was performed using an Applied Biosystems 7300 Real-Time PCR system with StepOne software version 2.2.2 (Thermo Fisher Scientific). Data were analyzed by the $\Delta \mathrm{C}_{t}$ method with mRNA expression normalized to glyceraldehyde-3-phosphate dehydrogenase mRNA.

\section{Gene Silencing}

siGENOME nontargeting control siRNA and siGENOME SMARTpool Rictor-specific, SMAD2-specific, and SMAD4-specific siRNAs each containing four distinct oligos targeting different regions of Rictor, SMAD2, and SMAD4 were obtained from Dharmacon (Thermo Fisher Scientific). Transfections were performed for 48 hours using the Lipofectamine RNAiMAX Reagent (Invitrogen). Silencing was confirmed by immunoblot analysis.

\section{Enzyme-Linked Immunosorbant Assay}

Activated TGF- $\beta 1$, secreted into the cell culture supernatant, was detected by means of an enzyme-linked immunosorbent assay, as per the manufacturer's instructions (Quantikine Human TGF $\beta-1$ Immunoassay; R\&D Systems, Minneapolis, $\mathrm{MN}$ ). The results were normalized to the total protein content of the corresponding cell culture lysates, as determined by the Bradford assay.

\section{Modified Scratch Wound Migration Assay}

Modified scratch wound migration assays were performed as previously described. ${ }^{31}$ Briefly, tissue culture plates were pretreated with $20 \mathrm{ng} / \mathrm{mL}$ fibronectin in phosphate-buffered saline for 1 hour. A thin strip of polydimethylsiloxane was placed in the middle of each well and allowed to adhere. Cells were seeded on top and incubated for 18 hours, after which the polydimethylsiloxane strips were removed to reveal an unperturbed fibronectin matrix. Multiple fields of view per well were imaged at regular intervals using the Leica DM IRE2 microscope (Leica, Buffalo Grove, IL) and MetaMorph imaging software version 7.8 (Molecular Devices, Sunnyvale, CA).

\section{Transwell Invasion Assay}

Transwell invasion assays were performed using polyester membranes ( $8 \mu \mathrm{m}$ thick; Corning Life Sciences, Tewksbury, MA) coated with Matrigel (BD Biosciences). Cells were seeded onto membranes in serum-free medium and allowed to migrate for 24 hours toward a paired chamber containing medium supplemented with $5 \mathrm{ng} / \mathrm{mL}$ TGF- $\beta$. Membranes were fixed with $4 \%$ paraformaldehyde, permeabilized in $0.1 \%$ Triton X-100, and stained with DAPI. The top of the membrane was wiped clean with a cotton swab so that only 

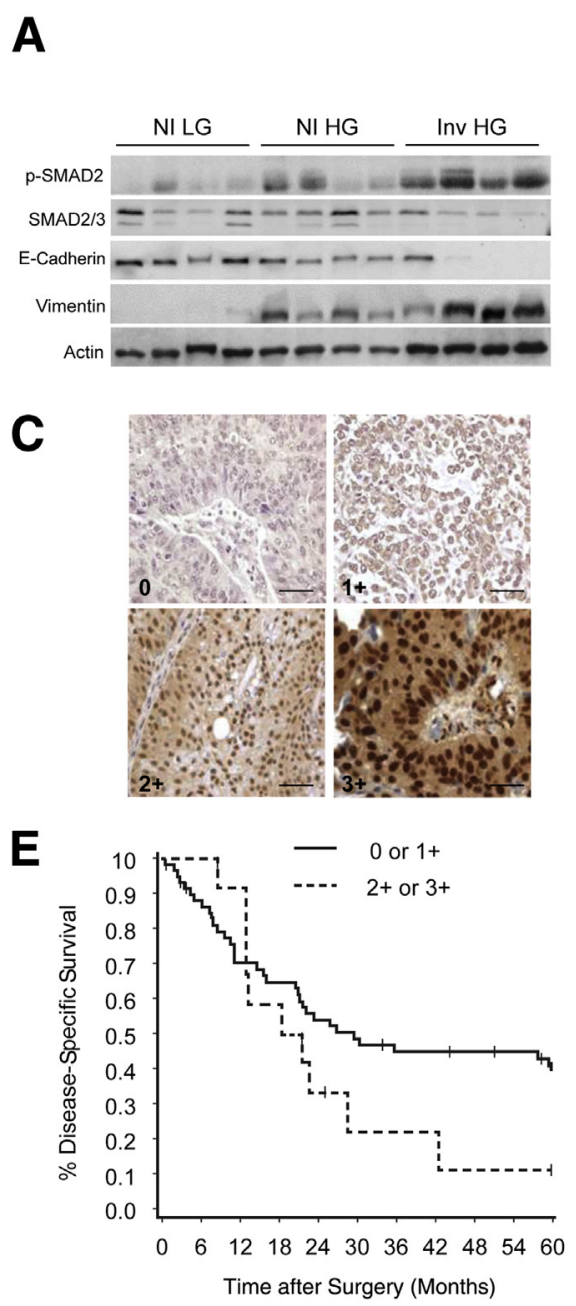
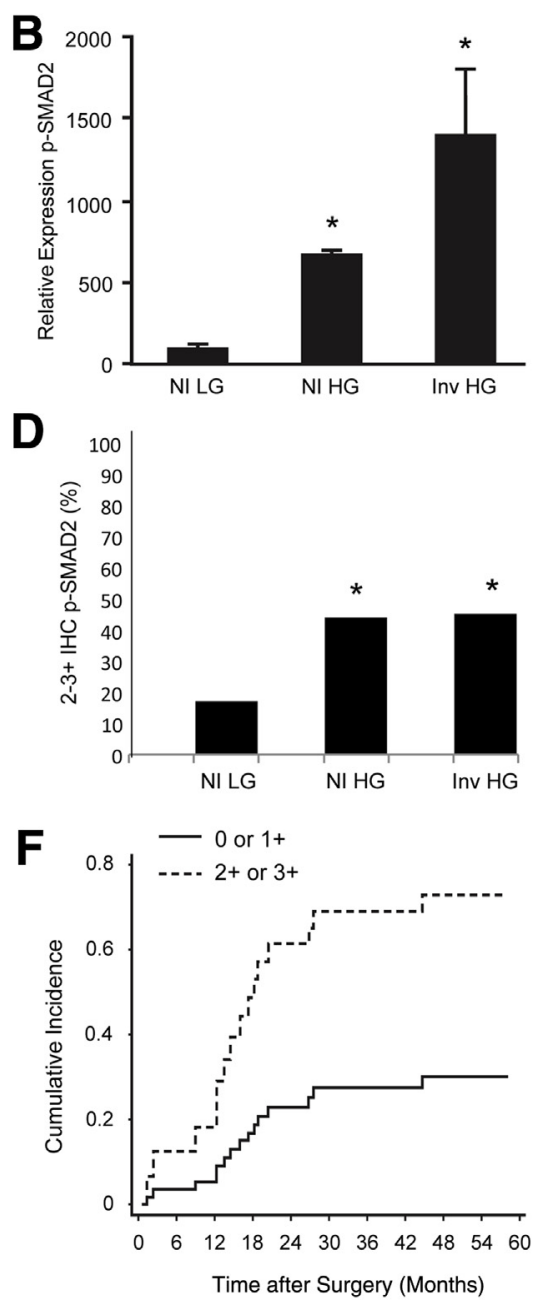

Figure 1 SMAD2 phosphorylation is increased in advanced bladder cancer and associated with diminished outcomes. A: Immunoblotting for p-SMAD2 and Vimentin shows increased levels in muscle-invasive high-grade bladder cancer (inv HG) compared with both noninvasive low-grade (NI LG) and noninvasive high-grade (NI HG) papillary carcinomas. B: Quantification of immunoblots shows a significant increase in p-SMAD2 levels in NI HG and inv HG lesions relative to $L G$ samples (percentage change standardized to total SMAD2/3). C: Representative images of $p$ SMAD2 immunohistochemical staining. D: Increased p-SMAD2 (2 to 3+immunostaining) was identified in high-grade bladder cancer and was associated with metastases in the invasive cohort. Increased $p$ SMAD2 is associated with decreased disease-specific survival $(P=0.02 ; \mathbf{E})$ and increased cumulative incidence $(P=0.01 ; \mathbf{F})$ in the invasive bladder cancer cohort. ${ }^{*} P<0.05$. Scale bar $=40 \mu \mathrm{m}$ (C). transmigrating cells were counted. The entire field of view was imaged at $\times 200$ magnification using the Leica DMR microscope and the number of cells present was quantified using ImageJ software version 1.47 (NIH, Bethesda, MD).

\section{Data Extraction from the Cancer Genome Atlas Project}

Data from the Cancer Genome Atlas project related to bladder cancer were mined via the publically available cBioPortal. ${ }^{32-34}$ We explored the following genes for mutation status, copy number alteration, and mRNA expression: TGFB1, TGFB2, TGFB3, TGFBR1, TGFBR2, SMAD1-7, SMAD9, BMP1-7, BMP8B, GDF2, BMP10, PTEN, PIK3CA, AKT1, AKT2, AKT3, TSC1, TSC2, MTOR, TP53, RB1, ARIDIA, KDM6A, and KMT2C.

\section{Results}

Phosphorylation of SMAD2 Is Increased in High-Grade Bladder Cancer and Associated with Reduced Survival and Increased Recurrence

We tested human bladder cancer specimens to determine whether TGF- $\beta$ activity, as assessed by phosphorylation of
SMAD2 (p-SMAD2), was altered in bladder cancers of different grades and stage. Immunoblotting analysis for p-SMAD2 was performed on representative human samples of noninvasive low-grade papillary urothelial carcinoma, noninvasive high-grade papillary urothelial carcinoma, and muscleinvasive high-grade urothelial carcinoma (Figure 1A). Our results show an increase in p-SMAD2 expression in high-grade bladder cancer, with highest levels in the invasive subset. Transition from E-cadherin to Vimentin expression was also seen with increasing grade and stage. Densitometric quantification of the ratio of $\mathrm{p}-\mathrm{SMAD} 2 /$ total SMAD2/3 confirmed these findings (Figure 1B).

Immunohistochemical analysis of p-SMAD2, an indicator of TGF- $\beta$ activity, was performed on 141 specimens from patients with noninvasive low-grade (36 patients), noninvasive high-grade (32 patients), or invasive (73 patients) urothelial carcinoma. Nuclear p-SMAD2 expression was scored from 0 (no expression) to $3+$ (strong expression) (Figure 1C). Using a cutoff of 2 to $3+$ immunoreactivity, an approximate 2.5 -fold increase in p-SMAD2 was seen in patients with either noninvasive or invasive high-grade disease relative to noninvasive low-grade disease (Figure 1D). In patients with invasive disease, elevated levels of p-SMAD2 expression were 

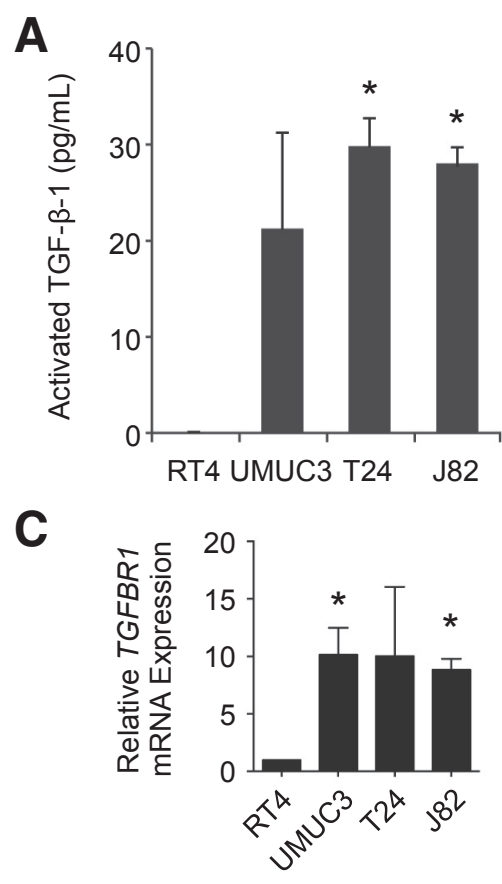

$\mathbf{E}$

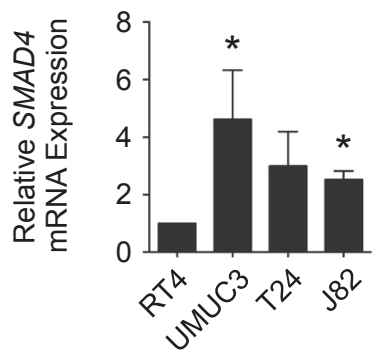

B

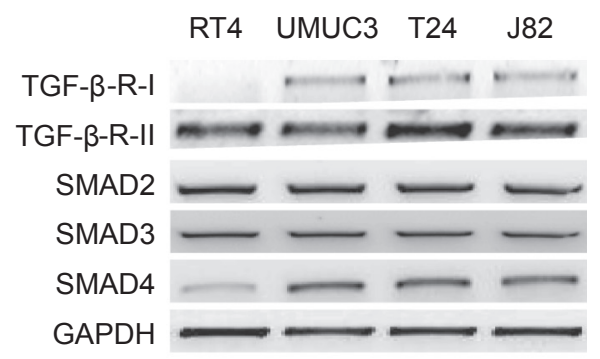

D

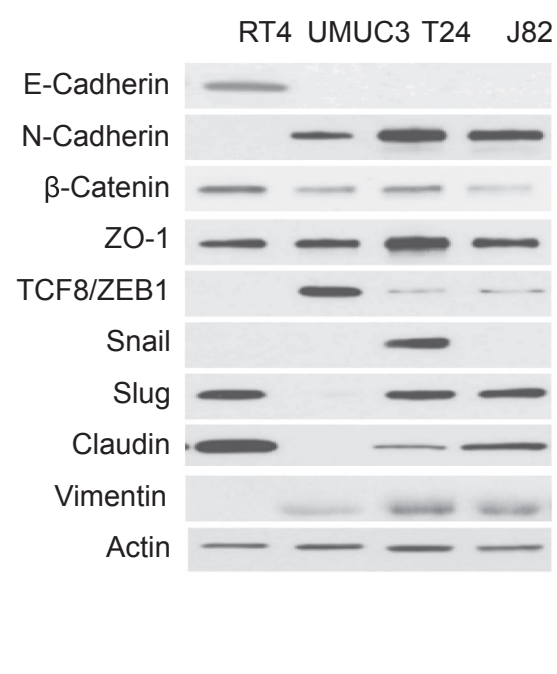

Figure 2 Transforming growth factor (TGF)- $\beta$ production and mesenchymal phenotype is increased in the J82, T24, and UMUC3 bladder cell lines. A: An enzyme-linked immunosorbent assay was performed to show secretion of activated TGF- $\beta$ into the cell culture medium. B: RT-PCR for TGF- $\beta$ receptor isoforms and activating SMADs in RT4, UMUC3, T24, and J82 bladder cancer cell lines. C: Real-time PCR for TGFBR1. D: Immunoblotting for markers of epithelialmesenchymal transition shows complimentary profiles in RT4 versus UMUC3, J82, and T24 bladder cells that was unchanged by $5 \mathrm{ng} / \mathrm{mL}$ TGF- $\beta$ application to RT4 cells or serum starvation of invasive cells. E: Realtime PCR for SMAD4. * $P<0.05$. GAPDH, glyceraldehyde-3-phosphate dehydrogenase; TCF8, transcription factor 8; ZEB1, zinc finger E-box-binding homeobox $1 ;$ Z0-1, zonula occludens protein 1 . independently associated with reduced disease-specific survival $(P=0.02)$ (Figure $1 \mathrm{E})$ and increased cumulative incidence of disease $(P=0.01)$ (Figure $1 \mathrm{~F}$ ) when controlled for pathological stage, presence of carcinoma in situ, angiolymphatic invasion, and patient age.

\section{Invasive Bladder Cells Show Up-Regulation of TGF- $\beta$ Pathway Elements in Association with Increased Vimentin Expression}

Our laboratory has previously demonstrated that the highly motile urothelial carcinoma cell lines T24, J82, and UMUC3 have high levels of mTORC2 activity and invasive capacity compared with the more epithelioid and less invasive RT4 bladder cancer cell line. ${ }^{7} \mathrm{We}$ assessed the conditioned media from these cell lines for TGF- $\beta$ secretion using enzymelinked immunosorbent assay and showed significant levels of secreted TGF- $\beta$ by T24, J82, and UMUC3 cells compared with RT4 cells (Figure 2A). TGF- $\beta$ receptor isoforms and SMADs in these cell lines were next assessed using real-time PCR (Figure 2B), with increased expression of TGFBR1 and SMAD4 confirmed by real-time PCR (Figure 2, C and E). TGF- $\beta$ R1, which forms a heteromeric receptor complex with TGF- $\beta$ R2 to transduce TGF- $\beta$ signals, was expressed at low to absent levels in RT4 cells, whereas expression of receptor isoforms and SMAD 2, 3, and 4 were identified in all other cell lines. Because TGF- $\beta$ can induce EMT in some cancer models, we assessed the expression of epithelial and mesenchymal markers associated with EMT (E-cadherin, Ncadherin, $\beta$-catenin, zonula occludens protein 1 , TCF8/Zeb1, Snail, Slug, Claudin, and Vimentin) in our cell lines both before and after TGF- $\beta$ application (Figure 2D). Although RT4 cells showed an epithelial phenotype associated with E-cadherin expression, the UMUC3, J82, and T24 cells showed a mesenchymal phenotype associated with Vimentin, N-cadherin, and TCF8/ZEB1 expression. This is consistent with previously published gene expression data sets that have been used to characterize these cell lines. ${ }^{35}$ However, some expression of pro-EMT markers, such as $\beta$-catenin, zonula occludens protein 1, Slug, and Claudin, was noted in RT4 cells despite the finding that these cells show limited to no migration in modified scratch assay and invasion into Matrigel. ${ }^{7}$ Bladder cells did not show any alterations in their EMT signature with TGF- $\beta$ treatment in the RT4 cells or prolonged serum starvation in UMUC3, T24, or J82 cells (data not shown), suggesting that the latter group can undergo TGF- $\beta$-induced motility in the background of established EMT signatures. 

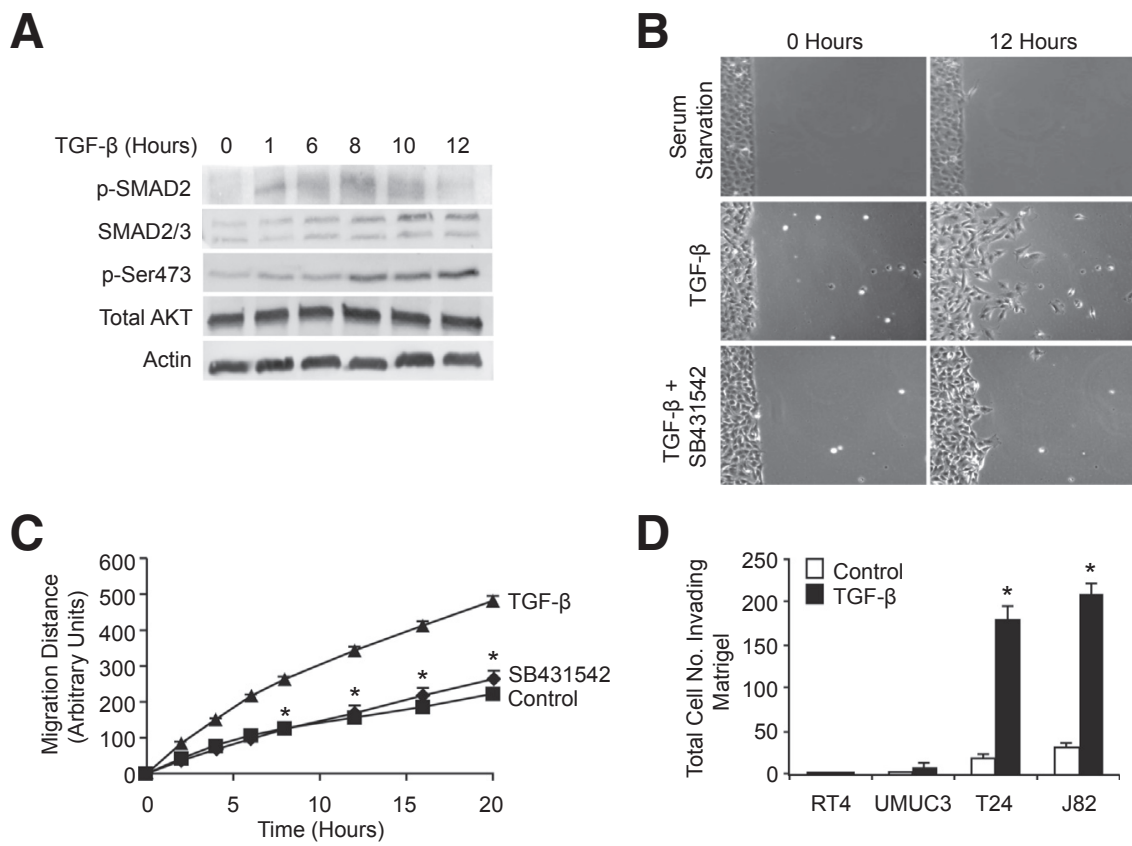

D

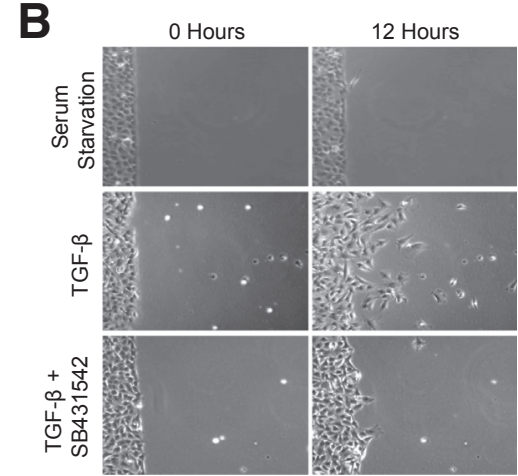

Figure 3 Transforming growth factor (TGF)- $\beta$ promotes cell motility and invasion in bladder cancer cells. A: TGF- $\beta$ induces mammalian target of rapamycin complex 2 (p-Ser473) activity at short and long time points. B: Time-lapse recording shows increased migration into the modified scratch wound (polydimethylsiloxane peel) field with TGF- $\beta$ application over 12 hours that can be ablated by the TFG- $\beta$ receptor inhibitor SB431542. C: Cumulative time course quantitation showing TGF- $\beta$-stimulated $\mathrm{J} 82$ motility that is blocked by application of SB431542. Serum starvation shows limited motility (control). D: TGF- $\beta$ induces Transwell invasion through Matrigel compared with untreated cells. ${ }^{*} P<0.05$.

\section{TGF- $\beta$ Induces mTORC2 Activity and Promotes Bladder Cancer Cell Migration and Invasion}

We have previously demonstrated that mTOR can influence bladder cancer cell migration and invasion, ${ }^{7,36}$ primarily through effects on mTOR complex 2 (mTORC2) induction of RhoA activity. ${ }^{7}$ Given the frequent alterations of TGF- $\beta$ components in advanced bladder cancer and the ability of TGF- $\beta$ to promote invasion in some cancer cell lines, we tested the ability of TGF- $\beta$ to mediate motility effects in bladder cancer. Application of TGF- $\beta$ to UMUC3, J82, and T24 bladder cancer cells increased p-SMAD2 levels within 1 hour, with sustained activity seen up to 10 hours (data shown only for J82 cells) (Figure 3A). TGF- $\beta$ also showed induction of mTORC2 activity, demonstrated by phosphorylation of the Ser473 residue of AKT (p-Ser473).

We next used a modified scratch wound assay, in which a polydimethylsiloxane membrane was used to generate a nondisrupted cell front, ${ }^{31}$ to test the impact of TGF- $\beta$ on bladder cancer cell migration (Figure $3 \mathrm{~B}$ ). In contrast to serum starvation conditions, TGF- $\beta$ increased the distance migrated by $\mathrm{J} 82$ cells. This effect was ablated by coincubation of cells with the TGF- $\beta R$ inhibitor SB431542. Quantification of time-lapse video microscopy of the migration front shows a persistent increase in cell migration with continuous TGF- $\beta$ exposure, suggesting that long-term cell modifications that influence migration could be occurring in these cells secondary to prolonged TGF- $\beta$ exposure (Figure $3 \mathrm{C}$ ). Similar changes were seen with the UMUC3 and T24 cell lines, though the extent of migration in TGF- $\beta$ stimulated UMUC3 cells was not statistically significant compared to serum starvation control conditions (data not shown). TGF- $\beta$ also increased invasion through Matrigel in the T24 and J82 cell lines (Figure 3D), but not UMUC3 cells.

\section{TGF- $\beta$-Mediated Bladder Cancer Cell Motility and Invasion Require mTORC2}

Given that mTORC2 is a critical mediator of migration and invasion in bladder cancer cells, we tested whether TGF$\beta$-induced motility is dependent on mTORC 2 signaling. TGF- $\beta$ application resulted in activation of mTORC2 (p-Ser473) signaling in T24 and J82 cells, with only mild effects on mTORC1 activity (Figure 4A). These effects were blocked by TGF- $\beta$ R inhibition using SB431542. RT4 cells showed minimal to no mTORC2 activity either at baseline or after TGF- $\beta$ stimulation, which likely is because of reduced TGF $\beta R I$ and SMAD4 expression in these cells. UMUC3 cells showed high baseline p-S6 and p-Ser473 levels even under serum starvation conditions (time 0), although a moderate reduction in mTORC 2 activity still occurred with TGF- $\beta$ R inhibition.

To test whether mTORC2 directly affects TGF$\beta$-induced bladder cell migration and invasion, we used selective silencing of Rictor, an essential mTORC2 component. Rictor silencing could be effectively achieved using pooled siRNA (Rictor siRNA), which resulted in absence of the Rictor protein and ablation of Ser473 AKT phosphorylation under both serum starvation and TGF- $\beta$ exposure conditions (Figure 4B). ${ }^{7}$ In contrast, control nontargeting pooled siRNA did not affect the ability of TGF- $\beta$ to activate mTORC2. Silencing of SMAD2 and SMAD4 did not ablate the ability of TGF- $\beta$ to increase Ser473 AKT phosphorylation (Figure 4C); however, SMAD2 silencing 
A

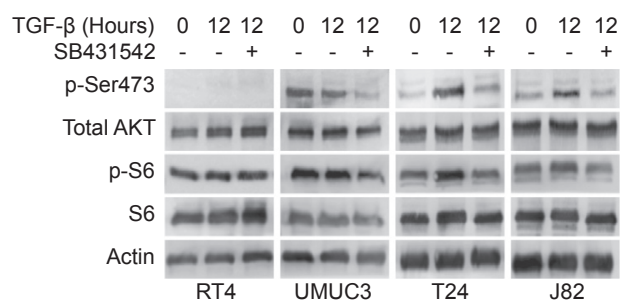

B

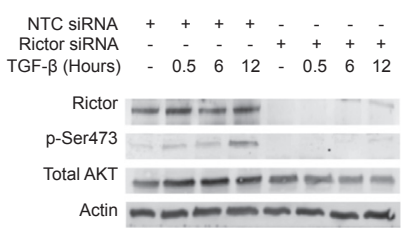

C
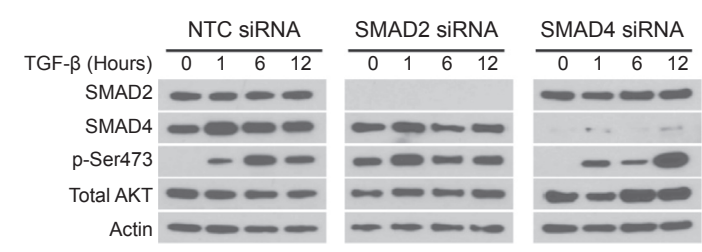

D

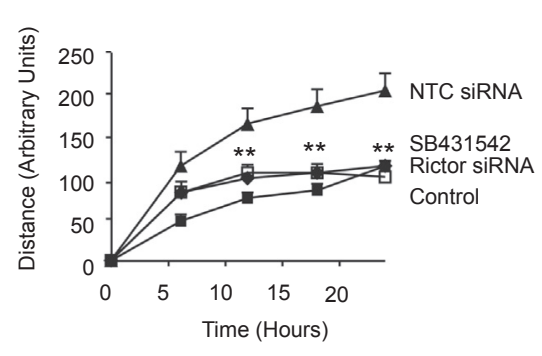

E

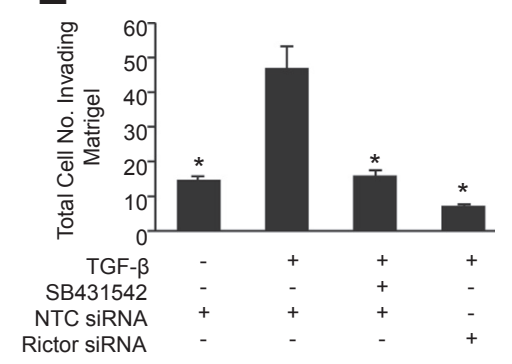

Figure 4 Transforming growth factor (TGF)- $\beta-$ driven motility and invasion requires mammalian target of rapamycin complex 2 (mTORC2). A: Immunoblot shows that TGF- $\beta$ induces both mTORC1 ( $p-S 6)$ and mTORC2 (p-Ser473) activity in J82 and T24 bladder cancer cell lines, whereas UMUC3 cells show a preexistent high baseline mTOR activity. SB431542 reduces mTORC1 and mTORC2 activity. B: Immunoblotting shows that TGF- $\beta$-induced phosphorylation of AKT Ser473 in $\mathrm{J} 82$ cells is inhibited by silencing of Rictor. C: Selective silencing of SMAD2 and SMAD4 still results in an induction of $\mathrm{p}$-Ser473 in response to TGF- $\beta$, although increased baseline $p$-Ser473 is present in SMAD2 silenced cells. D: J82 cells transfected with nontargeting control (NTC) siRNA increase motility in response to TGF- $\beta$ application on scratch wound assay compared with serumstarved cells transfected with NTC siRNA. Silencing of Rictor significantly reduces migration (pooled siRNA) and demonstrates similar effects to TGF- $\beta$ receptor-I inhibition with SB431542. A total of six separate frames were analyzed for each time point per condition. E: Transwell invasion assays with Matrigel show a significant reduction in invasion with Rictor silencing (Rictor siRNA) in malignant cells compared with TGF- $\beta$-treated cells transfected with NTC siRNA. Rictor silencing reduces invasion to levels equivalent to SB431542-treated cells. ${ }^{*} P<0.05,{ }^{*}{ }^{*} P<0.01$. also resulted in Ser473 AKT phosphorylation at baseline. Silencing of Rictor in both T24 and J82 cells ablated TGF$\beta$-induced cell migration in modified scratch wound assay that was equivalent to serum starved cells transfected with control siRNA and SB431452 treated control cells (Figure 4D). Nontargeting control siRNA-treated control cells showed persistent migration in response to TGF- $\beta$. Rictor silencing could also significantly reduce the ability of bladder cancer cells to invade through Matrigel (Figure 4E), suggesting that mTORC2 is critical in mediating TGF$\beta$-induced bladder cancer cell migration and invasion.

\section{TGF- $\beta$ and mTOR Pathway Component Alterations in Bladder Cancer}

Publicly available data from 131 patients available through cBioPortal were screened for gene amplification, deep deletions, missense and truncating mutations, and changes in mRNA expression in TGF- $\beta$ and mTOR pathway components (Figure 5, A and B). ${ }^{32-34}$ These samples were all collected from patients with at least muscle-invasive bladder cancer (pathological stage T2 or higher) with no prior systemic chemotherapy, per Cancer Genome Atlas Protocol. ${ }^{34}$ Most alterations represented either amplifications or mRNA up-regulation of TGF- $\beta$ isoforms and receptors, although several samples showed deep deletions of the bone morphogenetic protein family. Although most bladder cancers showed alterations in the mTOR signaling pathway, TGF- $\beta$ superfamily and receptor alterations were less common, suggesting that nongenomic mechanisms of increased TGF- $\beta$ signaling may be relevant in a subset of bladder cancer specimens.

\section{Discussion}

The extent of invasion into the bladder wall is a primary driver of diminished patient outcomes. ${ }^{3}$ However, the knowledge of the cellular mechanism(s) that influence this behavior in bladder cancer has not been fully elucidated. Our prior study has identified mTORC2 as an important regulator of invasive behavior in urothelial carcinoma of the bladder, ${ }^{7}$ and these findings have been supported by the results of the Cancer Genome Atlas project, which has identified frequent alterations of mTOR pathway components in advanced muscle-invasive bladder cancer. ${ }^{34} \mathrm{Up}$ stream activators of mTORC2 in bladder cancer, however, have not been well defined. We demonstrate a critical role for TGF- $\beta$-induced bladder cancer cell motility and invasion that is mediated through mTORC2 activity in this cancer system.

TGF- $\beta$ binds a heteromeric complex of TGF $\beta$ RI and TGF $\beta$ RII that results in phosphorylation of TGF $\beta$ RI and downstream phosphorylation of receptor-regulated SMAD2 and SMAD3. ${ }^{37}$ Once activated, these SMADs complex with 
A

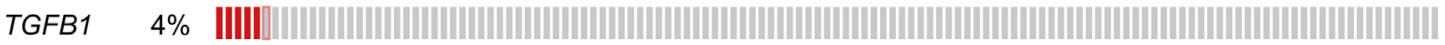

TGFB2 $4 \% \quad$ -

TGFB3 $12 \%$.

TGFBR1 $4 \%$.

TGFBR2 $12 \%$.

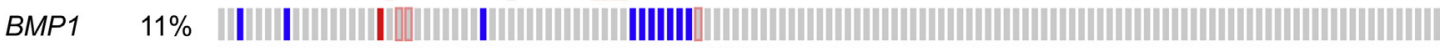

BMP2 $\quad 7 \%$.

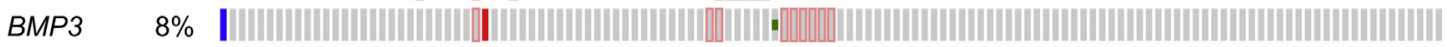

BMP4 $6 \%$.

BMP5 $\quad 7 \% \quad$ B.

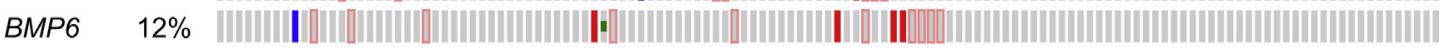

BMP7 $\quad 7 \%$.

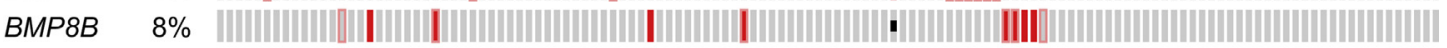

GDF2 $11 \%$ |

BMP10 $4 \%$ |

Genetic Alteration | Amplification | Deep Deletion I Missense Mutation I Truncating Mutation

\mRNA Up-Regulation

B

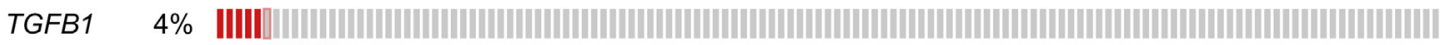

TGFB2 $4 \% \quad$ 4.

TGFB3 $12 \%$ 2.

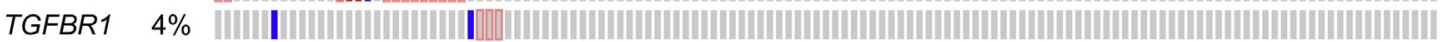

TGFBR2 $12 \%$.

SMAD1 $4 \%$.

SMAD2 $11 \%$.

SMAD3 $8 \% \quad$ ||

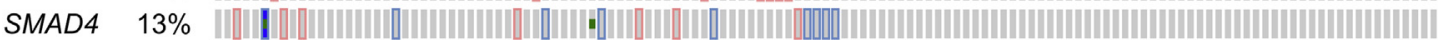

SMAD5 $5 \%$.

SMAD6 $2 \% \quad$ 2\%

SMAD7 $7 \%$.

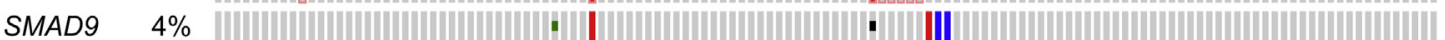

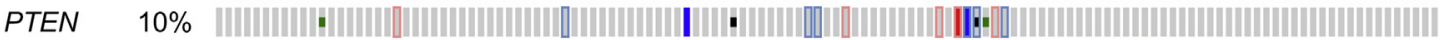

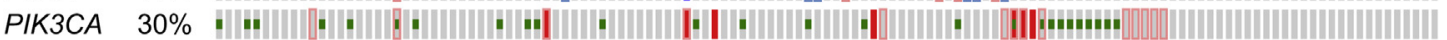

AKT1 $13 \%$.

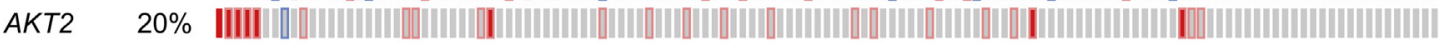

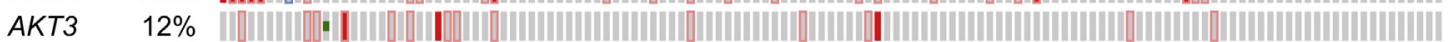

TSC1 $17 \%$ |

TSC2 $7 \%$.

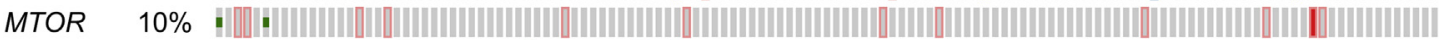

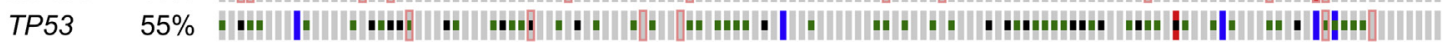

RB1 25\%

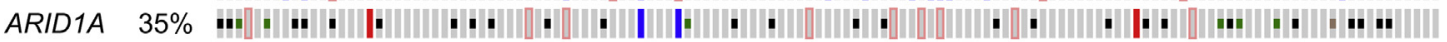

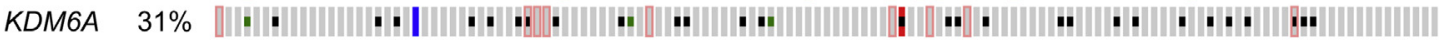

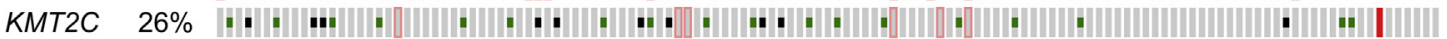

Genetic Alteration | Amplification | Deep Deletion I Missense Mutation I Truncating Mutation

॥ mRNA Up-Regulation || mRNA Down-Regulation

Figure 5 A: Transforming growth factor (TGF)- $\beta$ superfamily alterations in bladder cancer. B: DNA and RNA alterations of the mTOR pathway occur frequently in bladder cancer, with less frequent alteration in TGF- $\beta$ pathway members. The commonly altered TP53, RB1, ARID1A, KDM6A, and KMT2C genes are shown and do not appear to selectively associate with TGF- $\beta$ pathway alterations.

SMAD4 and translocate to the nucleus to increase transcription of target genes. ${ }^{38,39}$ Comparison to human samples that contained a progression spectrum of grade and stage confirmed that phosphorylation of SMAD2 was increased in advanced lesions. A similar result was seen in our cell lines, in which the high-grade bladder cancer cells UMUC3, T24, and J82 showed TGF- $\beta$ pathway activation relative to the more epithelioid RT4 bladder cancer cells, which lacked 
critical elements, such as the TGF- $\beta R 1$ isoform and SMAD4, and which expressed E-cadherin. Despite the various genomic alterations affecting the mTOR pathway in these cell lines, TGF- $\beta$ still retained the capacity to increase mTORC2 signaling. Given that the mTOR pathway is associated with numerous genomic alterations in human bladder cancer and is likely activated in most bladder cancers, ${ }^{34}$ our findings suggest that TGF- $\beta$ may influence mTOR signaling at least in the context of mTORC2-mediated migration and invasion. It is likely that other canonical and noncanonical signaling effects of TGF- $\beta$ can occur in bladder cancer cells, especially given that SMAD2 can influence baseline mTORC2 activity and may affect the activity of mTORC1. Prior studies by our laboratory have shown a critical role for mTORC1 activity in bladder cancer cell proliferation and survival, rather than invasion, ${ }^{36}$ although the role of TGF- $\beta$ in this context is not known.

TGF- $\beta$ has been previously shown to promote the epithelial-to-mesenchymal transition as part of the EMT process, ${ }^{12,13}$ with recent data suggesting that phosphatidylinositol 3-kinase-AKT-mTOR signaling may be critical in some cells to regulate this transition. ${ }^{14,40}$ In our human tissue samples, TGF- $\beta$ activity did appear to increase before the onset of EMT, which also was associated with increased mTORC 1 and mTORC 2 activity in these same samples, as reported in a prior publication from our laboratory. ${ }^{7}$ However, our cell line studies demonstrated a stable mesenchymal phenotype that was not reversed with prolonged TGF- $\beta$ withdrawal or with Rictor silencing (data not shown). These results still could suggest that TGF- $\beta$ induction of EMT may occur earlier in the bladder cancer neoplastic process, such as during the development of high-grade neoplasia, thus enabling cells to develop motile capacity secondary to other stimuli.

Although TGF- $\beta$ application did not induce EMT, exposure of bladder cancer cells to TGF- $\beta$ still induced motility and invasive behavior. Effects on mTOR signaling and motility in this paradigm were seen at both short-term and extended time points. The latter finding suggests that additional secondary effects of TGF- $\beta$, possibly including SMAD-induced transcriptional events or mTORC1-driven translation, are occurring that could promote motility and invasion in bladder cancer cells.

The association between phosphorylated SMAD2 and reduced disease-specific survival and time to recurrence has not been previously reported in bladder cancer and may represent a useful biomarker in the context of progression, EMT, and mTOR activation. One exception that may be of clinical relevance is the $10 \%$ of bladder cancers that show PTEN gene alterations, which in vitro correspond to high baseline mTOR activity unresponsive to TGF- $\beta$. Development of personalized therapies in this context requires further exploration.

In summary, our study shows that TGF- $\beta$ can induce bladder cancer cell motility and invasion with a requirement for mTORC2 activity. Further studies that evaluate the role of TGF- $\beta$ in early bladder neoplasia are needed to determine the influence of TGF- $\beta$ in establishment of a mesenchymal
EMT phenotype. Because of the relatively high incidence of bladder cancer in the population, further studies that test the functional impact of cooperative TGF- $\beta$ and mTOR signaling, as well as the role of phosphorylated SMAD2 as a novel biomarker, may provide unique opportunities to expand prognostic therapeutic paradigms in bladder cancer.

\section{Acknowledgments}

We thank Dr. Philip Howe (Medical University of South Carolina, Charleston, SC) for providing transforming growth factor- $\beta 2$.

\section{References}

1. American Cancer Society: Cancer Facts \& Figures 2014. Atlanta, American Cancer Society, 2014

2. Lotan Y, Kamat AM, Porter MP, Robinson VL, Shore N, Jewett M, Schelhammer PF, deVere White R, Quale D, Lee CT: Key concerns about the current state of bladder cancer: a position paper from the Bladder Cancer Think Tank, the Bladder Cancer Advocacy Network, and the Society of Urologic Oncology. Cancer 2009, 115:4096-4103

3. Tumours of the urinary system. Pathology and Genetics of Tumours of the Urinary System and Male Genital Organs. Edited by Eble JN, Sauter G, Epstein JI, Sesterhenn IA. World Health Organization Classification of Tumours. Lyon, France: IARC Press, 2004, pp. 90-157

4. Ching CB, Hansel DE: Expanding therapeutic targets in bladder cancer: the PI3K/Akt/mTOR pathway. Lab Invest 2010, 90:1406-1414

5. Gulhati P, Bowen KA, Liu J, Stevens PD, Rychahou PG, Chen M, Lee EY, Weiss HL, O'Connor KL, Gao T, Evers BM: mTORC1 and mTORC2 regulate EMT, motility, and metastasis of colorectal cancer via RhoA and Rac1 signaling pathways. Cancer Res 2011, 71:3246-3256

6. Masri J, Bernath A, Martin J, Jo OD, Vartanian R, Funk A, Gera J: mTORC2 activity is elevated in gliomas and promotes growth and cell motility via overexpression of rictor. Cancer Res 2007, 67: $11712-11720$

7. Gupta S, Hau AM, Beach JR, Harwalker J, Mantuano E, Gonias SL, Egelhoff TT, Hansel DE: Mammalian target of rapamycin complex 2 (mTORC2) is a critical determinant of bladder cancer invasion. PLoS One 2013, 8:e81081

8. Zinzalla V, Stracka D, Oppliger W, Hall MN: Activation of mTORC2 by association with the ribosome. Cell 2011, 144:757-768

9. Laplante M, Sabatini DM: mTOR signaling in growth control and disease. Cell 2012, 149:274-293

10. Pickup M, Novitskiy S, Moses HL: The roles of TGFbeta in the tumour microenvironment. Nat Rev Cancer 2013, 13:788-799

11. Yang L, Pang Y, Moses HL: TGF-beta and immune cells: an important regulatory axis in the tumor microenvironment and progression. Trends Immunol 2010, 31:220-227

12. Thiery JP, Sleeman JP: Complex networks orchestrate epithelialmesenchymal transitions. Nat Rev Mol Cell Biol 2006, 7:131-142

13. Zavadil J, Bottinger EP: TGF-beta and epithelial-to-mesenchymal transitions. Oncogene 2005, 24:5764-5774

14. Lamouille S, Connolly E, Smyth JW, Akhurst RJ, Derynck R: TGFbeta-induced activation of mTOR complex 2 drives epithelialmesenchymal transition and cell invasion. J Cell Sci 2012, 125: $1259-1273$

15. Vo BT, Morton D Jr, Komaragiri S, Millena AC, Leath C, Khan SA: TGF-beta effects on prostate cancer cell migration and invasion are mediated by PGE2 through activation of PI3K/AKT/mTOR pathway. Endocrinology 2013, 154:1768-1779

16. Castillejo A, Rothman N, Murta-Nascimento C, Malats N, GarciaClosas M, Gomez-Martinex A, Lloreta J, Tardon A, Serra C, 
Garcia-Closas R, Chanock S, Silverman DT, Dosemeci M, Kogevinas M, Carrato A, Soto JL, Real FX: TGFB1 and TGFBR1 polymorphic variants in relationship to bladder cancer risk and prognosis. Int J Cancer 2009, 124:608-613

17. Chen T, Jackson C, Costello B, Singer N, Colligan B, Douglass L, Pemberton J, Deddens J, Graff JR, Carter JH: An intronic variant of the TGFBR1 gene is associated with carcinomas of the kidney and bladder. Int J Cancer 2004, 112:420-425

18. Wei H, Kamat AM, Aldousari S, Ye Y, Huang M, Dinney CP, Wu X: Genetic variations in the transforming growth factor beta pathway as predictors of bladder cancer risk. PLoS One 2012, 7:e51758

19. Coombs LM, Pigott DA, Eydmann ME, Proctor AJ, Knowles MA: Reduced expression of TGF beta is associated with advanced disease in transitional cell carcinoma. Br J Cancer 1993, 67:578-584

20. Miyamoto H, Kubota Y, Shuin T, Torigoe S, Dobashi Y, Hosaka M: Expression of transforming growth factor-beta 1 in human bladder cancer. Cancer 1995, 75:2565-2570

21. Izadifar V, de Boer WI, Muscatelli-Groux B, Maille $P$, van der Kwast TH, Chopin DK: Expression of transforming growth factor beta1 and its receptors in normal human urothelium and human transitional cell carcinomas. Hum Pathol 1999, 30:372-377

22. Lee DH, Yang SC, Hong SJ, Chung BH, Chung HJ, Tokunaga H, Kim IY, Song YS, Lerner SP, Morton RA Jr: The loss of expression of transforming growth factor-beta receptors correlates with the histopathologic tumor grade in bladder transitional cell carcinoma patients. Yonsei Med J 1999, 40:118-123

23. Kim JH, Shariat SF, Kim IY, Menesses-Diaz A, Tokunaga H, Wheeler TM, Lerner SP: Predictive value of expression of transforming growth factor-beta(1) and its receptors in transitional cell carcinoma of the urinary bladder. Cancer 2001, 92:1475-1483

24. Tokunaga H, Lee DH, Kim IY, Wheeler TM, Lerner SP: Decreased expression of transforming growth factor beta receptor type I is associated with poor prognosis in bladder transitional cell carcinoma patients. Clin Cancer Res 1999, 5:2520-2525

25. McGarvey TW, Tait E, Tomaszewski JE, Malkowicz SB: Expression of transforming growth factor-beta receptors and related cell-cycle components in transitional-cell carcinoma of the bladder. Mol Urol 1999, 3:371-380

26. Song K, Shankar E, Yang J, Bane KL, Wahdan-Alaswad R, Danielpour D: Critical role of a survivin/TGF-beta/mTORC1 axis in IGFI-mediated growth of prostate epithelial cells. PLoS One 2013, 8:e61896

27. Zhang GJ, Ladehoff D, Xu Y, See WA: Stable overexpression of TGFbeta1 in a transitional carcinoma cell line: impact on fibronectin production. J Urol 1998, 160:230-235

28. Champelovier P, El Atifi M, Mantel F, Rostaing B, Simon A, Berger F, Seigneurin D: In vitro tumoral progression of human bladder carcinoma: role for TGFbeta. Eur Urol 2005, 48:846-851
29. Dehnavi E, Soheili ZS, Samiei S, Ataei Z, Aryan H: The effect of TGF-beta2 on MMP-2 production and activity in highly metastatic human bladder carcinoma cell line 5637. Cancer Invest 2009, 27: $568-574$

30. Li Y, Yang K, Mao Q, Zheng X, Kong D, Xie L: Inhibition of TGFbeta receptor I by siRNA suppresses the motility and invasiveness of T24 bladder cancer cells via modulation of integrins and matrix metalloproteinase. Int Urol Nephrol 2010, 42:315-323

31. Beach JR, Hussey GS, Miller TE, Chaudhury A, Patel P, Monslow J, Zheng Q, Keri RA, Reizes O, Bresnick AR, Howe PH, Egelhoff TT: Myosin II isoform switching mediates invasiveness after TGF-betainduced epithelial-mesenchymal transition. Proc Natl Acad Sci U S A 2011, 108:17991-17996

32. Gao J, Aksoy BA, Dogrusoz U, Dresdner G, Gross B, Sumer SO, Sun Y, Jacobsen A, Sinha R, Larsson E, Cerami E, Sander C, Schultz N: Integrative analysis of complex cancer genomics and clinical profiles using the cBioPortal. Sci Signal 2013, 6:pl1

33. Cerami E, Gao J, Dogrusoz U, Gross BE, Sumer SO, Aksoy BA, Jacobsen A, Byrne CJ, Heuer ML, Larsson E, Antipin Y, Reva B, Goldberg AP, Sander C, Schultz N: The cBio cancer genomics portal: an open platform for exploring multidimensional cancer genomics data. Cancer Discov 2012, 2:401-404

34. Cancer Genome Atlas Research Network: Comprehensive molecular characterization of urothelial bladder carcinoma. Nature 2014, 507: $315-322$

35. Lee JK, Havaleshko DM, Cho H, Weinstein JN, Kaldjian EP, Karpovich J, Grimshaw A, Theodorescu D: A strategy for predicting the chemosensitivity of human cancers and its application to drug discovery. Proc Natl Acad Sci U S A 2007, 104:13086-13091

36. Hansel DE, Platt E, Orloff M, Harwalker J, Sethu S, Hicks JL, DeMarzo A, Steinle RE, Hsi ED, Theodorescu D, Ching CB, Eng C: Mammalian target of rapamycin (mTOR) regulates cellular proliferation and tumor growth in urothelial carcinoma. Am J Pathol 2010, 176: 3062-3072

37. Massague J: TGFbeta signalling in context. Nat Rev Mol Cell Biol 2012, 13:616-630

38. Peinado H, Olmeda D, Cano A: Snail, Zeb and bHLH factors in tumour progression: an alliance against the epithelial phenotype? Nat Rev Cancer 2007, 7:415-428

39. Fuxe J, Vincent T, Garcia de Herreros A: Transcriptional crosstalk between TGF-beta and stem cell pathways in tumor cell invasion: role of EMT promoting Smad complexes. Cell Cycle 2010, 9: 2363-2374

40. Lamouille S, Derynck R: Emergence of the phosphoinositide 3-kinaseAkt-mammalian target of rapamycin axis in transforming growth factor-beta-induced epithelial-mesenchymal transition. Cells Tissues Organs 2011, 193:8-22 